\title{
Exploration of genetically encoded voltage indicators based on a chimeric voltage sensing domain
}

\author{
Yukiko Mishina ${ }^{1,2}$, Hiroki Mutoh ${ }^{1,3}$, Chenchen Song ${ }^{4}$ and Thomas Knöpfel ${ }^{1,4}$ * \\ ${ }^{1}$ Laboratory for Neuronal Circuit Dynamics, RIKEN Brain Science Institute, Wako, Japan \\ ${ }^{2}$ Centre for Global Communication Strategies, The University of Tokyo, Tokyo, Japan \\ ${ }^{3}$ Department of Neurophysiology, Hamamatsu University School of Medicine, Shizuoka, Japan \\ ${ }^{4}$ Division of Brain Sciences, Department of Medicine, Imperial College London, London, UK
}

\section{Edited by:}

Katsuhiko Mikoshiba, RIKEN Brain

Science Institute, Japan

\section{Reviewed by:}

Manuel L. Covarrubias, Thomas Jefferson University, USA

Alexandre Mourot, Université Pierre et Marie Curie, France

\section{*Correspondence:}

Thomas Knöpfel, Division of Brain Sciences, Department of Medicine, Imperial College London,

Hammersmith Hospital Campus, Du Cane Road, London W12 ONN, UK e-mail: t.knopfel@imperial.ac.uk
Deciphering how the brain generates cognitive function from patterns of electrical signals is one of the ultimate challenges in neuroscience. To this end, it would be highly desirable to monitor the activities of very large numbers of neurons while an animal engages in complex behaviors. Optical imaging of electrical activity using genetically encoded voltage indicators (GEVIs) has the potential to meet this challenge. Currently prevalent GEVIs are based on the voltage-sensitive fluorescent protein (VSFP) prototypical design or on the voltage-dependent state transitions of microbial opsins. We recently introduced a newVSFP design in which the voltage-sensing domain (VSD) is sandwiched between a fluorescence resonance energy transfer pair of fluorescent proteins (termed VSFP-Butterflies) and also demonstrated a series of chimeric VSD in which portions of the VSD of Ciona intestinalis voltage-sensitive phosphatase are substituted by homologous portions of a voltage-gated potassium channel subunit. These chimeric VSD had faster sensing kinetics than that of the native Ci-VSD. Here, we describe a new set of VSFPs that combine chimeric VSD with the Butterfly structure. We show that these chimeric VSFP-Butterflies can report membrane voltage oscillations of up to $200 \mathrm{~Hz}$ in cultured cells and report sensory evoked cortical population responses in living mice. This class of GEVIs may be suitable for imaging of brain rhythms in behaving mammalians.

Keywords: optogenetics, mouse model, optical imaging, voltage imaging, FRET sensors

\section{INTRODUCTION}

Deciphering how the brain generates cognitive function from patterns of electrical signals is one of the ultimate challenges in neuroscience. Advances toward this goal require a better understanding of the "neuronal code," and being able to monitor electrical signals of very large neuronal populations with fine temporal resolution is central to the progress. Even when restricted to relatively simple behaviors (such as goal-directed motor actions in response to sensory stimuli), observing only cortical activities, and in small animals such as mice, the monitoring of electrical activities must cover mesoscopic areas of tissue (dimensions measured at the millimeter scale). Optical voltage imaging methods have, at least in principle, the spatio-temporal resolution necessary for this endeavor (Ross et al., 1974; Grinvald et al., 1977; Grinvald and Hildesheim, 2004). In particular, voltage-sensitive dyes have been fruitfully employed in widefield epifluorescence imaging (Shoham et al., 1999; Petersen et al., 2003a; Grinvald and Hildesheim, 2004), and this approach has contributed much to the understanding of cortical circuit dynamics, especially in visual and somatosensory areas (Shoham et al., 1999; Kenet et al., 2003; Petersen et al., 2003a,b; Grinvald and Hildesheim, 2004).

Recently developed genetically-encoded voltage indicators (GEVIs) promise to improve upon classical voltage-sensitive dyes in at least four aspects: (i) they allow for non-invasive transcranial imaging in species with thin craniums (such as mice), which eliminates the previously compulsory craniotomies for dye staining; (ii) they provide reliable recordings from the same neuronal population in a subject over prolonged periods of time for multiple sessions; (iii) they genetically target specific cell populations, so the signals originate only from specific neurons of interest in an otherwise diverse population; (iv) they enable transgenic expression strategies that provide highly reproducible expression of protein indicators in different animals to eliminate between-subject variability.

There are two classes of conceptual designs of GEVIs currently being pursued. The first type is the microbial opsin-based GEVIs that exhibit voltage-dependent state-transitions in their photocycles (Kralj et al., 2012; Maclaurin et al., 2013). These opsin-based probes were initially limited by their low brightness (Mutoh et al., 2012; Mutoh and Knöpfel, 2013), but this issue has been successfully addressed in very recent work (Gong et al., 2014; Zou et al., 2014). The second type is the voltage-sensitive fluorescent protein (VSFP) class of GEVIs. These utilize the voltage-dependent structural rearrangement of voltage-sensing domains (VSDs), which are homologous to the S1-S4 transmembrane segment of Kv potassium channels. Thus far, several VSFP derivatives have enabled voltage imaging in brain slices as well as in intact mouse brain (Akemann et al., 2010, 2012; Mutoh et al., 2012; St-Pierre et al., 2014).

The first VSFPs (VSFP1 and VSFP2.x) exploited the voltagedependent VSD structural rearrangement to modulate fluorescence resonance energy transfer (FRET) efficacy between a tandem 
pair of fluorescent proteins (Sakai et al., 2001; Dimitrov et al., 2007). Dissection of a FRET independent component of the voltage response led to the development of the monochromatic (single fluorescent protein) VSFP3.x (Lundby et al., 2008; Perron etal., 2009a,b). More recently, we introduced VSFPButterflies, in which two fluorescent proteins are positioned so that the VSD is now sandwiched between the FRET pair (Akemann et al., 2012). These VSFP-Butterflies permitted imaging of sub-threshold activity in vivo in specific neuronal populations in awake behaving mice (Akemann et al., 2012). The VSFP2.x, VSFP3.x, and the VSFP-Butterfly scaffolds were adopted for other fluorescent proteins (Tsutsui et al., 2008, 2013; Jin et al., 2012).

The first VSFP with robust signals in mammalian cells used the voltage sensor of Ciona intestinalis voltage-sensitive phosphatase (Ci-VSP) whose VSD is homologous to that of Kv potassium channels (VSFP2.1; Dimitrov et al., 2007). Subsequent VSFP type of GEVIs [e.g., VSFP2.3 and VSFP3.1 (Lundby et al., 2008); VSFP2.4 (Akemann et al., 2010); VSFP-mUKG-mKOк (Tsutsui et al., 2008); VSFP-CR (Lam et al., 2012); ArcLight (Jin et al., 2012)], and ASAP1 (St-Pierre et al., 2014) generally substituted different fluorescent proteins or VSDs and varied the linking arrangements of the two components.

In order to overcome the limited response kinetics of current VSFPs, we developed chimeric VSDs in which portions of the Ci-VSP VSD was replaced by homologous portions of the Kv3.1 voltage-gated potassium channel subunit (Mishina et al., 2012). Insertion of these chimeric VSDs into the VSFP2.3 scaffold led to a series of chimeric VSFP variants, many of which efficiently target to the membrane of PC12 and human embryonic kidney (HEK) cells and exhibit optimized kinetics which retained Kv3.1 characteristics.

Here, we describe a new set of VSFPs that combine the chimeric VSDs with the VSFP-Butterfly structure. We show that these chimeric VSFP-Butterflies can report membrane voltage oscillations of up to $200 \mathrm{~Hz}$ in cultured cells and report sensory evoked cortical population responses in living mice. These variants of GEVIs may be suitable for imaging of brain rhythms in awake, behaving mammals.

\section{MATERIALS AND METHODS MOLECULAR BIOLOGY}

The chimeric Butterfly constructs were based on previously published versions of VSFPs, namely a combination of Chimera C5 (Mishina et al., 2012), in which a region of the VSD of CiVSP was substituted with that of the Kv3.1 potassium channel and VSFP-Butterfly 1.2 (Akemann et al., 2012; Figure 1). Both Chimeric VSFP-Butterfly cyan-yellow (CY; mCerulean/mCitrine) and Chimeric VSFP-Butterfly yellow-red (YR; mCitrine/mKate2) were generated using sequential polymerase chain reactions following the previously published protocols (Lundby et al., 2008; Mutoh et al., 2009; Akemann et al., 2012; Mishina et al., 2012). Briefly, Chimeric VSFP-Butterfly YR was generated by substituting the Ci-VSP VSD sequence of VSFP-Butterfly 1.2 (Akemann etal., 2012) with that of Kv3.1 VSD. This was performed by introducing restriction sites (XhoI and EcoRV) at the terminal ends of the VSD in both VSFP-Butterfly 1.2 and Chimera
A

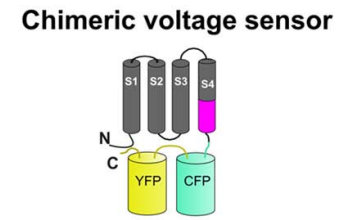

Ci-VSP

mKv3.1 Chimera C5
RLVVLARLLRVVRLARIFYSHQQM 240 RVVRFVRILRIFRLTRHFVGLRVL $\mathbf{3 3 4}$ RLVVLARLLRIFKLTRHFVGHQQM 240
B

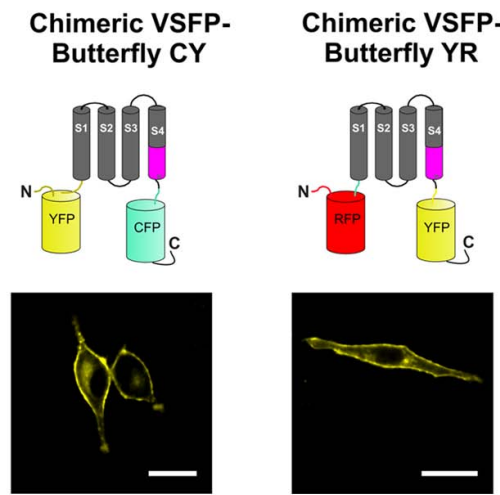

FIGURE 1 | Design of a chimeric voltage sensor by transferring a fragment of Kv3.1 to Ciona intestinalis voltage-sensitive phosphatase (Ci-VSP) in the S4 transmembrane segment. (A) Schematic depiction (top) of a chimeric voltage sensor, Chimera $\mathrm{C} 5$, in which a section of the voltage-sensing domain (VSD) of Ci-VSP is exchanged with amino acids from the S4 transmembrane segment of Kv3.1 (bottom). The fluorescent proteins that are attached to the $\mathrm{C}$-terminus of the chimeric construct enables visualization of membrane targeting and monitoring of voltage-dependent fluorescence changes due to movements of the VSD. (B) Modifications in the pair of the fluorescent proteins and localization of the constructs at the plasma membrane. The donor fluorescent protein was kept at the C-terminus, whereas the acceptor fluorescence was attached to the $\mathrm{N}$-terminus. The donor/acceptor pairs were $\mathrm{mCerulean/mCitrine} \mathrm{for}$ Chimeric voltage-sensitive fluorescent protein (VSFP)-Butterfly cyan-yellow (CY; left) and mCitrine/mKate2 for Chimeric VSFP-Butterfly yellow-red (YR; right). Scale bar, $20 \mu \mathrm{m}$.

C5 (Mishina etal., 2012) as silent mutations and substituting the Chimera C5 VSD into the VSFP-Butterfly 1.2. In addition, a single mutation, K234R of mKate2, was introduced by site-directed mutagenesis for decreased intracellular aggregation and enhanced brightness (Perron and Knöpfel, unpublished observations). Chimeric VSFP-Butterfly CY was designed to incorporate the mCerulean/mCitrine fluorescence reporters, rather than the $\mathrm{mCitrine} / \mathrm{mKate}$ pair (Mutoh et al., 2009). Similar to the VSFP-Butterfly 1.0 (Akemann et al., 2012), the mCitrine FRET acceptor was attached to the VSD at position 70 by overlap extension polymerase chain reactions after removal of the mCitrine of VSFP2.3. All constructs were subsequently subcloned into both pcDNA3.1(-; for functional imaging in cell culture) and pCAG vectors (for in vivo imaging; Lundby etal., 2008; Akemann etal., 2012) by utilizing NheI and AflII restriction endonucleases. DNA sequences for all of the constructs were confirmed by DNA sequencing analysis. 
Chimeric VSFP-Butterfly CY and chimeric VSFP-Butterfly YR are deposited at Addgene (pCAG-Chimeric_Butterfly_CY_1.0, 59800; pCAG-Chimeric_Butterfly_YR_1.0, 59801).

\section{CELL CULTURE, IN VITRO OPTICAL IMAGING, AND IN UTERO ELECTROPORATION}

PC12 cells were cultured in Dulbecco's modified Eagle's medium supplemented with $10 \%$ horse serum, $5 \%$ fetal bovine serum, and $1 \%$ penicillin and streptomycin (GIBCO) at $37^{\circ} \mathrm{C}$. HEK293T cells were cultured in Dulbecco's modified Eagle's medium supplemented with $10 \%$ fetal bovine serum and $1 \%$ penicillin and streptomycin (GIBCO) at $37^{\circ} \mathrm{C}$. Cells were grown on poly-Dlysine coated coverslips and transfected $24 \mathrm{~h}$ after plating using Lipofectamine 2000 reagent (Invitrogen) and washed daily. Experiments were performed 2-3 days after transfection. PC12 cell images were obtained with a confocal laser scanning microscope (C1si/FN1, Nikon) for expression screening. In utero electroporation were performed as previously described (Akemann et al., 2010).

\section{ELECTROPHYSIOLOGY AND FUNCTIONAL OPTICAL IMAGING}

The voltage clamp recordings were performed on the instrumental set-up as previously described (Akemann et al., 2010, 2012). Briefly, voltage-dependent fluorescence recordings from both PC12 and HEK cells were performed by combining voltage clamp (under the whole-cell configuration of the patch-clamp technique) with dual-emission microfluorometry. Electrical and optical data were acquired using pCLAMP 10.1 software (Axon Instruments). PC12 or HEK cells were continuously perfused $(1.5-2 \mathrm{ml} / \mathrm{min}$ ) with a bathing solution containing (in $\mathrm{mM}$ ) $150 \mathrm{NaCl}, 4 \mathrm{KCl}, 2 \mathrm{CaCl}_{2}, 1 \mathrm{MgCl}_{2}, 5$ Glucose, 5 HEPES (pH 7.4 with $\mathrm{NaOH})$. Patch electrodes had resistances of 3$5 \mathrm{M} \Omega$ when filled with intracellular solution containing (in $\mathrm{mM}$ ) $130 \mathrm{CsCl}, 1 \mathrm{MgCl}_{2}, 20$ HEPES, 5 EGTA, 3 MgATP (pH 7.2 with $\mathrm{CsOH}$ ). All data were low-pass filtered with a cutoff frequency of $5 \mathrm{kHz}$ and digitized at $5 \mathrm{kHz}$ using a Digidata 1322 analog-to-digital converter (Axon Instruments). Fluorescence was illuminated by light from a computer-controlled monochromator (Polychrome IV, T.I.L.L. Photonics). For VSFP2.3, Chimera C5 and Chimeric VSFP-Butterfly CY, excitation light (440 nm) was reflected and first passed through a 458-nm dichroic mirror (FF458-Di01, Semrock). Emitted fluorescence was then split by a 506-nm dichroic mirror (FF506-Di03, Semrock) onto two photodiodes (T.I.L.L. Photonics) behind Cerulean- and Citrine- specific filters (BP $482 \pm 35 \mathrm{~nm}$ : FF01-482/35-25 and LP 514 nm: LP02-514RU-25, Semrock). For Chimeric VSFPButterfly YR, excitation light $(488 \mathrm{~nm})$ was reflected and first passed through a 506-nm dichroic mirror (FF506-Di03, Semrock). Emitted fluorescence was then split by a $593-\mathrm{nm}$ dichroic mirror (FF593-Di03, Semrock) onto two photodiodes (T.I.L.L. Photonics) behind Citrine- and mKate2- specific filters (BP $542 \pm 13.5 \mathrm{~nm}$ : FF01-542/27-25 and LP 594 nm: BLP01-594R-25, Semrock).

The following protocol was used to test the voltage-dependence of the constructs (Figure 2). From a holding potential of $-60 \mathrm{mV}$, cells were held for $500 \mathrm{~ms}$ at voltages between $-140 \mathrm{mV}$ and $120 \mathrm{mV}$ in $20 \mathrm{mV}$ steps to elicit fluorescence signals from
mCerulean and mCitrine (VSFP2.3, Chimera C5, and Chimeric VSFP-Butterfly CY) or mCitrine and mKate2 (Chimeric VSFPButterfly YR). Finally, to test the frequency response of the constructs, sinusoidal voltage oscillations ( $20 \mathrm{mV}$ amplitude) from -70 were generated in voltage clamp mode at frequencies of 10 , 50, 100, and $200 \mathrm{~Hz}$.

For all optical data, background data were obtained from a region on the coverslips devoid of fluorescent proteins. Photobleaching was corrected by division of a double exponential fit of the fluorescence trace at the holding potential. The ratiometric fluorescent signals were obtained by dividing the signals from the two fluorescent proteins. Calculations, including time constants, dynamic range, and $\mathrm{V}_{1 / 2}$ were calculated as previously described (Akemann et al., 2012). Data are expressed as mean \pm SEM, with $n$ specifying the number of independent experiments. For each cell and voltage protocol 6-10 traces were averaged.

In vivo optical imaging was performed on mice (over 60 days old) in utero electroporated with expression plasmids. A glass window was implanted under surgical anesthesia (pentobarbital by intraperitoneal injection) as previously described (Akemann et al., 2012). Briefly, after exposing the cranial bone by removing the soft tissue, the left somatosensory cortex was carefully thinned using a dental drill and a cover glass was mounted over the cortex. A metal bolt was fixed on the frontal-medial cranium with dental cement. For imaging, mice were mounted onto a custom-made stereotaxic frame for stabilization and the body temperature was kept at $37^{\circ} \mathrm{C}$ (Fine Science Tools, Tokyo). The stimulus to the contralateral whisker $\mathrm{C} 1$ was delivered by a focal air puff system (100 ms, Picospritzer III, Parker Hannifin). Dual-emission optical imaging was performed using two synchronized CCD cameras (Sensicam, PCO) at 50 frames/s. Excitation light was provided by a high-power halogen lamp (Moritex). The following filters and splitters were used for optical recording: mCitrine excitation (FF01 483/32-25), mCitrine emission (F01 542/27-25), mKate2 emission (BLP01 594R-25), LP506 nm (FF 506-Di03) as excitation beam splitter, and LP593 nm (FF 593-Di03) as detection splitter, all installed onto the THT macroscopy system (Brainvision, Tokyo). Images were acquired using a custom-made macros using ImagePro6.2.

Animal experiments were performed under the Institutional Animal Care and Use Committee of the RIKEN Wako Research Center.

\section{RESULTS \\ CHIMERIC VSFP-BUTTERFLIES}

We previously systematically replaced portions of the Ci-VSP VSD with homologous sections of the Kv3.1 potassium channel subunit, yielding chimeric VSDs that we termed Chimera $\mathrm{Cx}$ (x running from 1 to 40 ) and demonstrated that this replacement leads to an acceleration of voltage sensing movements in a subgroup of constructs (Mishina et al., 2012). For the present study, we used the construct Chimera $\mathrm{C} 5$, in which 10 consecutive amino acid segments from the C-terminal portion of the S4 of Kv3.1 replaced the homologous region in VSFP2.3 (Figure 1A).

The choice of the fluorescent protein pair for FRET measurements and the positioning relative to the VSD can 

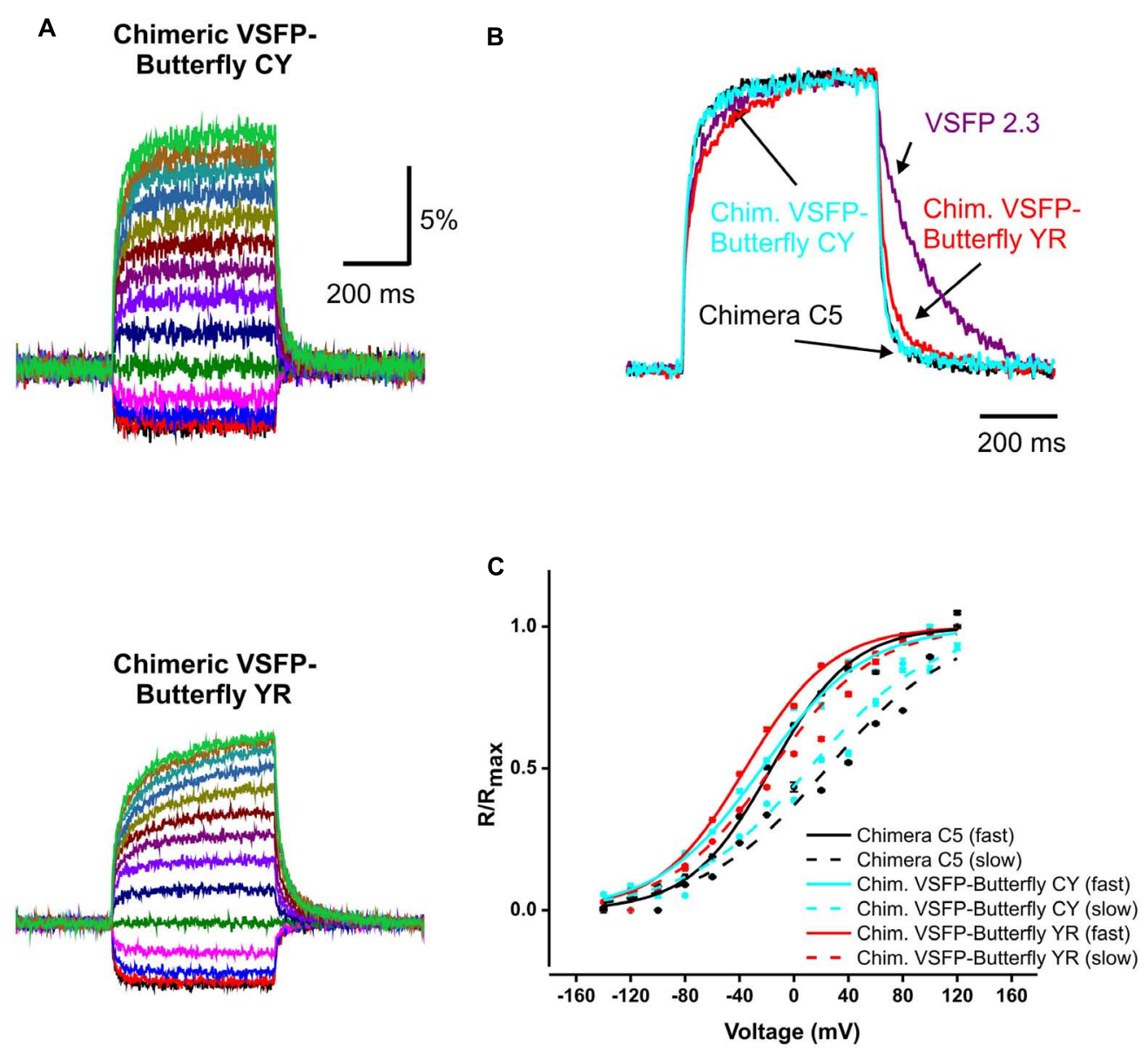

FIGURE 2 | Kinetics of voltage dependency of Chimeric VSFP-Butterfly CY and Chimeric VSFP-Butterfly YR in PC12 cells. (A) Comparison of ratiometric fluorescence signals of Chimeric VSFP-Butterfly $C Y$ (upper) and Chimeric VSFP-Butterfly YR (lower) in response to a family of voltage steps from a holding potential of $-60 \mathrm{mV}$, and $20 \mathrm{mV}$ step increments from -140 to $120 \mathrm{mV}$. $(n=6)$. (B) Normalized responses to depolarization from -60 to $20 \mathrm{mV}$ and responses to return for VSFP2.3 (magenta), Chimera C5 (black), Chimeric VSFP-Butterfly CY (blue), and Chimeric VSFP-Butterfly YR (red).
(C) Amplitude of normalized optical signal $(\Delta R / R)$ versus membrane voltage for the fast (solid line) and slow (dotted line) components of Chimera C5 (black), Chimeric VSFP-Butterfly CY (blue), and Chimeric VSFP-Butterfly YR (red). Responses $(\triangle R / R)$ to families of voltage steps (as in $\mathbf{A})$ were fitted with the function $y=y 0+A 1^{*} \exp \left(-t / \tau 1 \_o n\right)+A 2^{*} \exp \left(-t / \tau 2 \_o n\right)$ where $t$ is time, $\tau 1$ on the faster on time constant and $\tau 2$ _on the slower on time constant. A1 and $\mathrm{A} 2$ are the amplitudes of fast and slow response components and are plotted against the step voltage. $N=6-7$; error bars \pm SEM.

Table 1 | Summary of the FRET response properties of chimeric voltage-sensitive fluorescent protein (VSFP)-Butterflies and previously published VSFPs.

\begin{tabular}{|c|c|c|c|c|c|c|c|}
\hline & $\tau_{1}$ on & $\tau_{2}$ on & $\% \tau_{1}$ & $\tau$ off & $\mathrm{V}_{1 / 2}$ fast (mV) & $\mathrm{V}_{1 / 2}$ slow $(\mathrm{mV})$ & $\Delta \mathrm{R} / \mathrm{R}(\%)^{\#}$ \\
\hline Chimeric VSFP-Butterfly cyan-yellow (CY) & $2.1 \pm 0.2$ & $36.7 \pm 1.1$ & 60.0 & $14.6 \pm 0.5$ & $-24.0 \pm 0.5$ & $\geq 20$ & $14.7 \pm 0.2$ \\
\hline Chimera $\mathrm{C} 5^{*}$ & $2.1 \pm 0.2$ & $36.8 \pm 0.9$ & 60.1 & $13.4 \pm 0.5$ & $-17.9 \pm 0.4$ & $\geq 20$ & $14.8 \pm 0.1$ \\
\hline VSFP2.3* & $3.0 \pm 0.2$ & $69.2 \pm 1.8$ & 26.6 & $91.6 \pm 1.7$ & $-28.3 \pm 0.5$ & $-48.6 \pm 0.5$ & $15.2 \pm 0.1$ \\
\hline
\end{tabular}

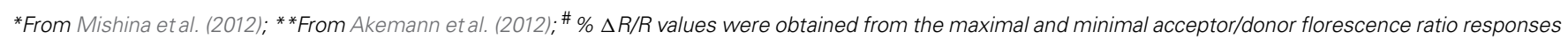
between -140 and $120 \mathrm{mV}$ from a holding potential of $-70 \mathrm{mV}$.

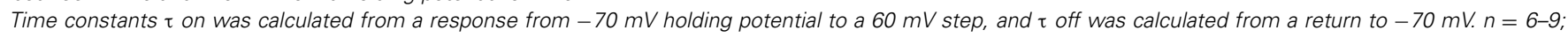
Errors \pm SEM. 
significantly affect the biophysical properties of FRET-based genetically encoded indicator proteins (Alford etal., 2013). By moving the FRET acceptor from the C-terminus of the donor to the N-terminal end of the VSD, we generated and characterized two new Butterfly variants of the Chimera C5 construct. The two Butterfly variants were designed with mCerulean/mCitrine ("CY") and mCitrine/mKate2 ("YR") fluorescent protein FRET pairs (Figure 1B) and they were named Chimeric VSFP-Butterfly CY and Chimeric VSFP-Butterfly YR, respectively.
We first tested whether the VSFP-Butterfly conformation preserved the kinetic properties found for Chimera C5 described in Mishina et al. (2012; Figure 2; Table 1). We found no significant ( $t$-test, $p=0.1-0.5$; Table 1) difference between chimera C5 and Chimeric VSFP-Butterfly CY in terms of the response time constants and the overall ratiometric signal amplitudes. The Chimeric VSFP-Butterfly YR version exhibited a slightly larger second on time constant and a slower off time constant when compared to Chimera C5 ( $t$-test, $p<0.001$ for both, Table 1).




One of the advantageous features of our previous VSFP Butterfly 1.2 was a left shifted $V_{1 / 2}$ value (as compared to its precursor VSFP2.42), increasing the sensitivity around the resting membrane potential (Akemann et al., 2012). A significant left shift of the $V_{1 / 2}$ values was found for Chimeric VSFP-Butterfly YR when compared to Chimera C5 ( $t$-test, $p<0.001$; Table 1). A clear improvement of the Chimeric VSFP-Butterfly YR as compared to VSFP Butterfly 1.2 was a significant shortening of the off time constant (25.1 \pm 0.9 versus $89.9 \pm 5.2 \mathrm{~ms}$; Akemann et al., 2012, $p<0.005)$. The faster off response was even more pronounced for Chimeric VSFP-Butterfly CY $(14.6 \pm 0.5$, significant smaller than the value of Chimeric VSFP-Butterfly YR, $t$-test, $p<0.001$; Table 1).

These characteristic parameters are summarized in Table 1 along with previous published measurements for VSFP2.3 (Lundby etal., 2008, 2010; Akemann etal., 2010) and VSFP Butterfly 1.2 (Akemann et al., 2012).

\section{RESPONSES OF THE CHIMERIC VSFP-BUTTERFLY CONSTRUCTS TO OSCILLATORY MEMBRANE VOLTAGES}

To evaluate the use of chimeric VSFP-Butterflies for the potential to image brain rhythms in behaving mammalians, optical responses to oscillatory membrane potential were studied. To this end, PC12 and HEK cells were voltage clamped with an oscillatory voltage command. These experiments show that chimeric VSFP-Butterflies follow sinusoidal membrane voltage oscillations of up to $200 \mathrm{~Hz}$, confirming the above initial assessment of these constructs demonstrating optimized kinetics relative to VSFP-Butterfly 1.2 (Akemann et al., 2012; Figure 3A). Compared to VSFP-Butterfly 1.2 (Akemann et al., 2012), chimeric VSFPButterflies show a larger gain (sensitivity) at frequencies between 10 and $200 \mathrm{~Hz}(p<0.05, t$-test; Figure 3). The less pronounced loss of gain observed with chimeric VSFP-Butterflies between steady state sensitivity and report of oscillatory membrane voltage

A
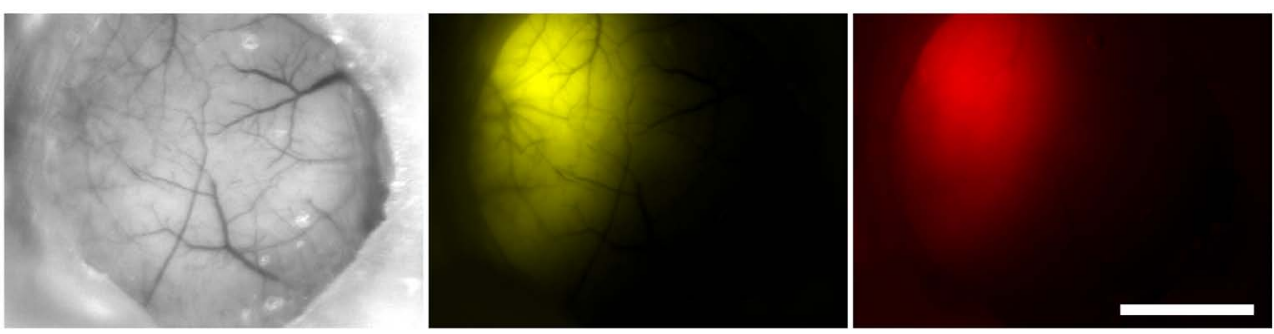

B
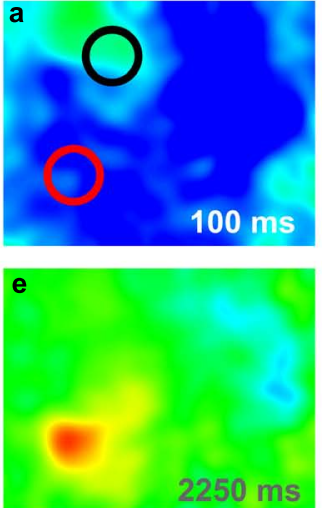
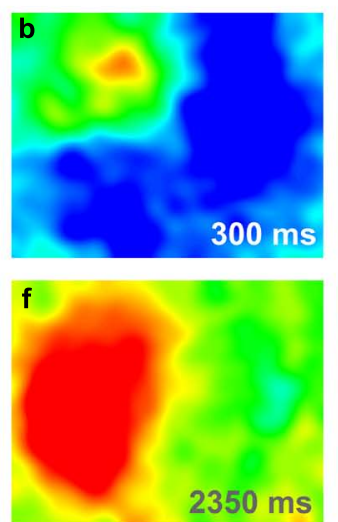

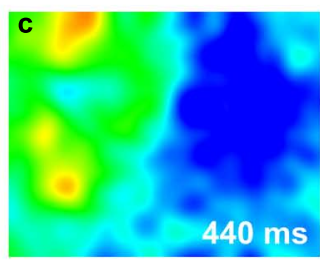

g

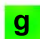

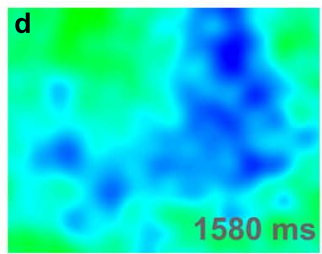

0.13

$\Delta \mathrm{R} / \mathrm{RO}(\%)$

0

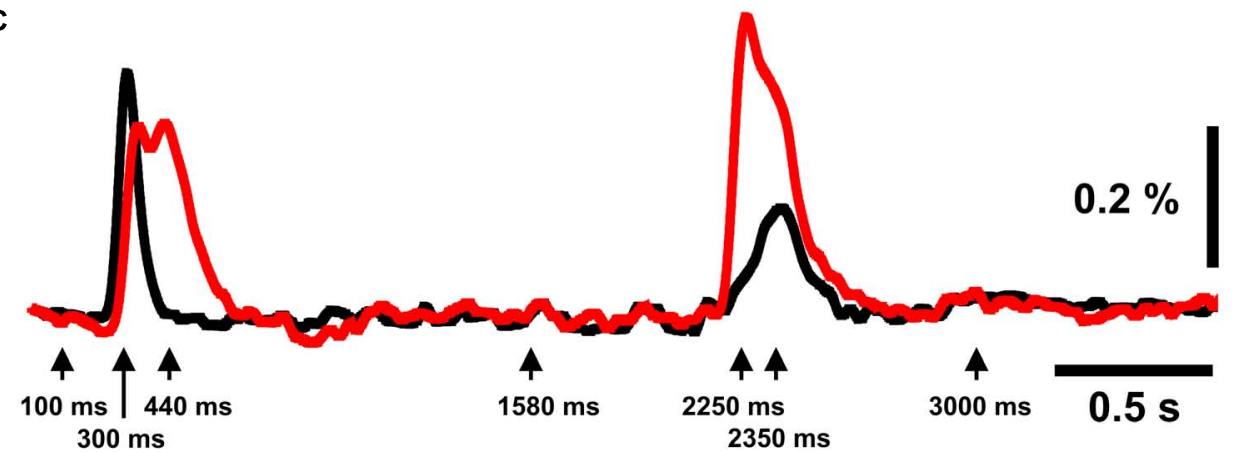

deflection of the $\mathrm{C} 1$ whisker and a brief flash of light. Scale bar, $2 \mathrm{~mm}$. (C) Black and red traces of the ratiometric signal are taken from the black (somatosensory cortex) and red (visual cortex) circles in (B-a), respectively. The arrows indicate the time points of the ratiometric images in (B). 
fluctuations (at $10 \mathrm{~Hz}$ or higher frequencies) can be explained by the larger contribution of the faster component of the on response $(\tau 1$ on) and the much faster off time constant ( $\tau 1$ off; Table 1).

\section{IN VIVO DEMONSTRATION OF THE FUNCTIONAL POTENTIALS OF THE CHIMERIC CONSTRUCTS}

Chimeric VSFP-Butterfly YR was taken as a representative chimeric construct to explore performance in vivo. A relatively large transcranial window covering the left hemisphere of mice electroporated with the Chimeric VSFP-Butterfly YR construct exhibited strong fluorescence signals (Figure 4A). Under anesthesia, the signal of the Chimeric VSFP-Butterfly YR on the somatosensory cortex could be induced from baseline (Figure $4 \mathrm{~B}-\mathrm{a}$ ) by a single C1 whisker deflection (Figure 4B-b) and the signal propagated to other discrete cortical areas (motor cortex and secondary somatosensory cortex, Figure 4B-c), and eventually faded (Figure 4B-d). Stimulation using brief flashes of light also activated the visual cortex area (Figure 4B-e) which propagated through the hemisphere toward the rostral area (Figure 4B-f) also then gradually faded (Figure 4B-g). Figure 4C shows how those voltage signals propagated in two distinct cortices, the somatosensory cortex and the visual cortex.

\section{DISCUSSION}

We generated and characterized a new set of VSFPs that combine a chimeric VSD with the VSFP-Butterfly structure. We show that these chimeric VSFP-Butterflies can report membrane voltage oscillations of up to $200 \mathrm{~Hz}$ in cultured cells. We also demonstrate that these GEVIs report sensory evoked cortical population responses in living mice. The in vivo assay is particularly important as some previously reported VSFP derivatives (e.g., "Mermaid") were functional in transfected cultured cells but failed to provide robust voltage signals in equivalent in vivo assays (Akemann et al., 2012). Similarly, it remains to be seen whether the recently reported monochromatic green fluorescent VSFPs with high in vitro sensitivity such as ASAP1 (St-Pierre et al., 2014) are suitable for rodent in vivo imaging experiments where modulation of green indicator fluorescence is significantly affected by hemodynamic effects (Diez-Garcia et al., 2007) and where movement artifacts are a major challenge.

The present study addresses the need for GEVIs with fast kinetics. Previous work has indicated that the kinetics of FRET-based VSFP is limited by properties of the voltage-sensitive protein that undergoes a slower state transition into a relaxed state after an initial, relatively fast on response (Lundby et al., 2010). To overcome this kinetic limit, VSDs from various species have also been explored. In particular, the chick homolog of Ci-VSP appears to provide a scaffold that lead to VSFPs with faster kinetics (Han et al., 2013; St-Pierre et al., 2014). The advantage of chimeras between $\mathrm{Ci}-\mathrm{VSP}$ and $\mathrm{Kv}$ potassium channel subunits, as introduced and employed here, is that $\mathrm{Kv}$ channels are among the best studied membrane proteins with a wealth of structural understanding (Villalba-Galea et al., 2008; Vargas et al., 2012). This will likely instigate a more rational approach in the fine-tuning of the voltage sensing portion of GEVIs.
Improvements of GEVIs will continue as they are essential tools in studies where processing of synaptic inputs and action potentials at frequencies greater than $10 \mathrm{~Hz}$ are of interest. We expect that voltage imaging will be instrumental when linking neuronal information flows across large cortical areas and complex behavior GEVIs are likely on their way to replace the current low molecular weight voltage-sensitive dyes used in such long-term mesoscopic (circuit-centric) imaging approaches (Knöpfel, 2012). In this brain mapping research domain, GEVIs will become central to bridging the gap between single-neuron and whole-brain recordings as they are able to target specific cell populations and are able to provide consistent recordings over extended periods at the same locations.

The new VSFPs reported here outperform previously published GEVIs with demonstrated in vivo performance that are based on coupling a VSD with a FRET pair of fluorescent proteins by a factor of two in response time while keeping former advantages and strengths such as utilizing ratiometric outputs and brightness necessary for robust, high-resolution signals. Future fine-tuning of the chimeric Butterflies introduced here may include further enhancements in VSD kinetics and dynamic range, and may involve varied fluorescent proteins for increased brightness and photostability.

\section{ACKNOWLEDGMENTS}

We would like to thank the members of the Knöpfel lab for scientific advice and insightful discussions. We also would like to thank GlaxoSmithKline for their financial support.

\section{REFERENCES}

Akemann, W., Mutoh, H., Perron, A., Park, Y. K., Iwamoto, Y., and Knöpfel, T. (2012). Imaging neural circuit dynamics with a voltage-sensitive fluorescent protein. J. Neurophysiol. 108, 2323-2337. doi: 10.1152/jn.00452.2012

Akemann, W., Mutoh, H., Perron, A., Rossier, J., and Knöpfel, T. (2010). Imaging brain electric signals with genetically targeted voltage-sensitive fluorescent proteins. Nat. Methods 7, 643-649. doi: 10.1038/nmeth.1479

Alford, S. C., Wu, J., Zhao, Y., Campbell, R. E., and Knöpfel, T. (2013). Optogenetic reporters. Biol. Cell 105, 14-29. doi: 10.1111/boc.201200054

Diez-Garcia, J., Akemann, W., and Knöpfel, T. (2007) In vivo calcium imaging from genetically specified target cells in mouse cerebellum. Neuroimage 34, 859-869. doi: 10.1016/j.neuroimage.2006.10.021

Dimitrov, D., He, Y., Mutoh, H., Baker, B. J., Cohen, L., Akemann, W., etal. (2007). Engineering and characterization of an enhanced fluorescent protein voltage sensor. PLoS ONE 2:e440. doi: 10.1371/journal.pone. 0000440

Gong, Y., Wagner, M. J., Zhong, L. J., and Schnitzer, M. J. (2014). Imaging neural spiking in brain tissue using FRET-opsin protein voltage sensors. Nat. Commun. 5, 3674. doi: 10.1038/ncomms4674

Grinvald, A., and Hildesheim, R. (2004). VSDI: a new era in functional imaging of cortical dynamics. Nat. Rev. Neurosci. 5, 874-885. doi: 10.1038/ nrn 1536

Grinvald, A., Salzberg, B. M., and Cohen, L. B. (1977). Simultaneous recording from several neurones in an invertebrate central nervous system. Nature 268, 140-142. doi: 10.1038/268140a0

Han, Z., Jin, L., Platisa, J., Cohen, L. B., Baker, B. J., and Pieribone, V. A. (2013). Fluorescent protein voltage probes derived from ArcLight that respond to membrane voltage changes with fast kinetics. PLOS ONE 8:e81295. doi: 10.1371/journal.pone.0081295

Jin, L., Han, Z., Platisa, J., Wooltorton, J. R., Cohen, L. B., and Pieribone, V. A. (2012). Single action potentials and subthreshold electrical events imaged in neurons with a fluorescent protein voltage probe. Neuron 75, 779-785. doi: 10.1016/j.neuron.2012.06.040 
Kenet, T., Bibitchkov, D., Tsodyks, M., Grinvald, A., and Arieli, A. (2003). Spontaneously emerging cortical representations of visual attributes. Nature 425, 954-956. doi: 10.1038/nature02078

Knöpfel, T. (2012). Genetically encoded optical indicators for the analysis of neuronal circuits. Nat. Rev. Neurosci. 13, 687-700. doi: 10.1038/nrn3293

Kralj, J. M., Douglass, A. D., Hochbaum, D. R., Maclaurin, D., and Cohen, A. E. (2012). Optical recording of action potentials in mammalian neurons using a microbial rhodopsin. Nat. Methods 9, 90-95. doi: 10.1038/nmeth.1782

Lam, A. J., St-Pierre, F., Gong, Y., Marshall, J. D., Cranfill, P. J., Baird, M. A., et al. (2012). Improving FRET dynamic range with bright green and red fluorescent proteins. Nat. Methods 9, 1005-1012. doi: 10.1038/nmeth.2171

Lundby, A., Akemann, W., and Knöpfel, T. (2010). Biophysical characterization of the fluorescent protein voltage probe VSFP2.3 based on the voltage-sensing domain of Ci-VSP. Eur. Biophys. J. 39, 1625-1635. doi: 10.1007/s00249-0100620-0

Lundby, A., Mutoh, H., Dimitrov, D., Akemann, W., and Knöpfel, T. (2008) Engineering of a genetically encodable fluorescent voltage sensor exploiting fast Ci-VSP voltage-sensing movements. PLoS ONE 3:e2514. doi: 10.1371/journal.pone.0002514

Maclaurin, D., Venkatachalam, V., Lee, H., and Cohen, A. E. (2013). Mechanism of voltage-sensitive fluorescence in a microbial rhodopsin. Proc. Natl. Acad. Sci. U.S.A. 110, 5939-5944. doi: 10.1073/pnas.1215595110

Mishina, Y., Mutoh, H., and Knöpfel, T. (2012). Transfer of Kv3.1 voltage sensor features to the isolated Ci-VSP voltage-sensing domain. Biophys. J. 103, 669-676. doi: 10.1016/j.bpj.2012.07.031

Mutoh, H., Akemann, W., and Knöpfel, T. (2012). Genetically engineered fluorescent voltage reporters. ACS Chem. Neurosci. 3, 585-592. doi: 10.1021/cn300041b

Mutoh, H., and Knöpfel, T. (2013). Probing neuronal activities with genetically encoded optical indicators: from a historical to a forward-looking perspective. Pflugers Arch. 465, 361-371. doi: 10.1007/s00424-012-1202-z

Mutoh, H., Perron, A., Dimitrov, D., Iwamoto, Y., Akemann, W., Chudakov, D. M., et al. (2009). Spectrally-resolved response properties of the three most advanced FRET based fluorescent protein voltage probes. PLoS ONE 4:e4555. doi: 10.1371/journal.pone.0004555

Perron, A., Mutoh, H., Akemann, W., Gautam, S. G., Dimitrov, D., Iwamoto, Y., etal. (2009a). Second and third generation voltage-sensitive fluorescent proteins for monitoring membrane potential. Front. Mol. Neurosci. 2:5. doi: 10.3389/neuro.02.005.2009

Perron, A., Mutoh, H., Launey, T., and Knöpfel, T. (2009b). Red-shifted voltage-sensitive fluorescent proteins. Chem. Biol. 16, 1268-1277. doi: 10.1016/j.chembiol.2009.11.014

Petersen, C. C., Grinvald, A., and Sakmann, B. (2003a). Spatiotemporal dynamics of sensory responses in layer $2 / 3$ of rat barrel cortex measured in vivo by voltagesensitive dye imaging combined with whole-cell voltage recordings and neuron reconstructions. J. Neurosci. 23, 1298-1309.

Petersen, C. C., Hahn, T. T., Mehta, M., Grinvald, A., and Sakmann, B. (2003b). Interaction of sensory responses with spontaneous depolarization in layer 2/3 barrel cortex. Proc. Natl. Acad. Sci. U.S.A. 100, 13638-13643. doi: $10.1073 /$ pnas. 2235811100
Ross, W. N., Salzberg, B. M., Cohen, L. B., and Davila, H. V. (1974). A large change in dye absorption during the action potential. Biophys. J. 14, 983-986. doi: 10.1016/S0006-3495(74)85963-1

Sakai, R., Repunte-Canonigo, V., Raj, C. D., and Knöpfel, T. (2001). Design and characterization of a DNA-encoded, voltage-sensitive fluorescent protein. Eur. J. Neurosci. 13, 2314-2318. doi: 10.1046/j.0953-816x.2001.01617.x

Shoham, D., Glaser, D. E., Arieli, A., Kenet, T., Wijnbergen, C., Toledo, Y., et al. (1999). Imaging cortical dynamics at high spatial and temporal resolution with novel blue voltage-sensitive dyes. Neuron 24, 791-802. doi: 10.1016/S0896-6273(00)81027-2

St-Pierre, F., Marshall, J. D., Yang, Y., Gong, Y., Schnitzer, M. J., and Lin, M. Z. (2014). High-fidelity optical reporting of neuronal electrical activity with an ultrafast fluorescent voltage sensor. Nat. Neurosci. 17, 884-889. doi: 10.1038/nn.3709

Tsutsui, H., Jinno, Y., Tomita, A., Niino, Y., Yamada, Y., Mikoshiba, K., et al. (2013). Improved detection of electrical activity with a voltage probe based on a voltage-sensing phosphatase. J. Physiol. 591, 4427-4437. doi: 10.1113/jphysiol.2013.257048

Tsutsui, H., Karasawa, S., Okamura, Y., and Miyawaki, A. (2008). Improving membrane voltage measurements using FRET with new fluorescent proteins. Nat. Methods 5, 683-685. doi: 10.1038/nmeth.1235

Vargas, E., Yarov-Yarovoy, V., Khalili-Araghi, F., Catterall, W. A., Klein, M. L., Tarek, M., et al. (2012). An emerging consensus on voltage-dependent gating from computational modeling and molecular dynamics simulations. J. Gen. Physiol. 140, 587-594. doi: 10.1085/jgp.201210873

Villalba-Galea, C. A., Sandtner, W., Starace, D. M., and Bezanilla, F. (2008). S4-based voltage sensors have three major conformations. Proc. Natl. Acad. Sci. U.S.A. 105, 17600-17607. doi: 10.1073/pnas.0807387105

Zou, P., Zhao, Y., Douglass, A. D., Hochbaum, D. R., Brinks, D., Werley, C. A., et al. (2014). Bright and fast multicoloured voltage reporters via electrochromic FRET. Nat. Commun. 5, 4625. doi: 10.1038/ncomms5625

Conflict of Interest Statement: The Associate Editor Katsuhiko Mikoshiba declares that, despite being affiliated to the same institution as authors, the review process was handled objectively and no conflict of interest exists. The authors declare that the research was conducted in the absence of any commercial or financial relationships that could be construed as a potential conflict of interest.

Received: 19 June 2014; accepted: 14 September 2014; published online: 29 September 2014.

Citation: Mishina Y, Mutoh H, Song C and Knöpfel T (2014) Exploration of genetically encoded voltage indicators based on a chimeric voltage sensing domain. Front. Mol. Neurosci. 7:78. doi: 10.3389/fnmol.2014.00078

This article was submitted to the journal Frontiers in Molecular Neuroscience. Copyright (c) 2014 Mishina, Mutoh, Song and Knöpfel. This is an open-access article distributed under the terms of the Creative Commons Attribution License (CC BY). The use, distribution or reproduction in other forums is permitted, provided the original author(s) or licensor are credited and that the original publication in this journal is cited, in accordance with accepted academic practice. No use, distribution or reproduction is permitted which does not comply with these terms. 